\title{
Along the Bos Taurus genome, uncover candidate Imprinting Control Regions
}

\author{
Phillip Wyss ${ }^{1}$, Carol Song ${ }^{2}$, and Minou Bina ${ }^{1}$ \\ ${ }^{1}$ Department of Chemistry, and ${ }^{2}$ Information Technology at Purdue University \\ West Lafayette, IN 47907 USA \\ Correspondence: Bina@Purdue.edu
}

\begin{abstract}
In mammals, Imprinting Control Regions (ICRs) regulate a subset of genes in a parent-of-origin-specific manner. In both human and mouse, previous studies identified a set of CpG-rich motifs that occurred as clusters in ICRs and germline Differentially Methylated Regions (gDMRs). These motifs consist of the ZFP57 binding site (ZFBS) overlapping a subset of MLL binding units known as MLL morphemes. Furthermore, by creating plots for displaying the density of these overlaps, it became possible to locate known and candidate ICRs in mouse and human genomic DNA. Since genomic imprinting impacts many developmental and key physiological processes, we performed genome-wide analyses to create plots displaying the density of the CpG-rich motifs (ZFBS-morph overlaps) along Bos Taurus chromosomal DNA. We tailored our datasets so that they could be displayed on the UCSC genome browser (the build bosTau8). On the genome browser, we could view the ZFP57 binding sites, the ZFBS-morph overlaps, and peaks in the density-plots in the context of cattle RefSeq Genes, Non-Cow RefSeq Genes, CpG islands, and Single nucleotide polymorphisms (SNPs). Our datasets revealed the correspondence of peaks in plots to known and deduced ICRs in Bos Taurus genomic DNA. We illustrate that by uploading our datasets onto the UCSC genome browser, we could discover candidate ICRs in cattle DNA. In enlarged views, we could pinpoint the genes in the vicinity of candidate ICRs and thus discover potential imprinted genes.
\end{abstract}

\section{BACKGROUND}

For centuries, breeders have relied on principles of inheritance to obtain animals with desired traits [1]. However, emerging data indicate that assisted reproductive technologies (ART) may induce fetal overgrowth, producing LOS -large offspring syndrome [1-7]. Partly, developmental anomalies arose from altered DNA methylation patterns causing imprinting defects in ICRs, candidate DMRs, or both [4, 5, 7-9]. Furthermore, researchers have observed that Somatic Cell Nuclear Transfer (SCNT) procedures, could epigenetically disturb imprinted gene expression [4]. 
Overall, genome imprinting is relatively complex and requires orchestrated action of several proteins, including ZFP57, KAP1, and a subset of DNA methyltransferases [10-13]. In ICRs/gDMRs, ZFP57 recognizes its methylated hexameric site [14] and thus plays a central role in the establishment of genomic imprints $[10,14,15]$. ZFP57 family members (KZFPs) are encoded in the hundreds by the genomes of higher vertebrates $[16,17]$. Most KZFPs are essential to the recruitment of KAP1 and associated effectors to chromatin to repress transcription [16]. ZFP57 is necessary to maintain the DNA methylation memory at multiple ICRs in mice embryos and embryonic stem cells $[10,14,15,18]$. In addition to ZFP57 binding sites, the ICRs in mouse often include closely-spaced ZFBS-morph overlaps [19]. These overlaps are composite-DNA-elements that could play dual but antagonistic roles in the regulation of allele-specific gene expression: ZFP57 binding to its methylated sites to maintain allele-specific gene repression; binding of MLL1 or MLL2 to CpG-rich sequences to protect ICRs from methylation to support transcription [19]. MLL1 (or MLL) is the founding member of a protein family with a domain for methylating lysine 4 in histone $\mathrm{H} 3$ producing $\mathrm{H} 3 \mathrm{~K} 4 \mathrm{me} 3$ marks in chromatin [20]. Among the family members, only MLL1/KMT2A and MLL2/KMT2B have the MT domain for binding unmodified CpG-rich DNA [21-23]. Furthermore, through association with several proteins, MLL contributes to the coordinated patterns of gene expression [24, 25].

In both mouse and human DNA, known ICRs/gDMRs encompassed clusters of two or more ZFBS-morph overlaps [26, 27]. Therefore, we wished to investigate whether known bovine ICRs also included these composite-DNA-elements for regulating parent-of-origin-specific expression. To do so, we performed genome-wide analyses of Bos Taurus chromosomal DNA sequences. Firstly, we located ZFP57 binding sites and ZFBS-morph overlaps in the sequences. Subsequently, we created density-plots to pinpoint ICRpositions in Bos Taurus DNA. By uploading our datasets onto the UCSC genome browser, we could obtain snapshots to view peak positions with respect to genomic landmarks. These snapshots uncovered a connection between peaks in plots and the ICRs in bovine imprinting domains including H19-IGF2, KCNQ1, PLAGL1, IGF2R, and PEG3. Additional snapshots revealed such connection for: the essential ICR in the GNAS complex locus; and intergenic ICRs in MEST, INPP5F, and MEG8 loci. Since peaks in plots could locate known ICRs/DMRs in bovine DNA, we anticipate that with our approach one could discover candidate ICRs and novel imprinted genes in cattle DNA.

\section{RESULTS}

For studies of genomic imprinting in cattle, we followed a previous approach applied to mouse and human genomic DNA [26, 27]. Briefly: at the UCSC genome browser, we retrieved the DNA sequences of each cattle chromosome (reported for the build bosTau8). Afterward, we wrote a Perl script to obtain the positions of the ZFP57 binding site and the ZFBS-morph overlaps in bovine DNA. Subsequently, we wrote another script to scan the file containing the positions of the ZFBS-morph overlaps to create density-plots. We tailored this script to count the number of ZFBS-morph overlaps in a sliding window, consisting of 850- 
bases, while omitting isolated occurrences. The output reported the midpoint for each window as the function of the number of overlap occurrences. We selected the window size by trial and error. Large windows tended to produce false peaks; small windows gave peaks with a spiky appearance. By uploading our datasets onto the UCSC genome browser, we created custom tracks to view the density-plots in the context of landmarks including the positions of genes, transcripts, the CpG islands, and SNPs. Furthermore, by using the browser tools, we could display peak positions in short DNA segments or along an entire chromosome. To evaluate the robustness of our strategy, the following sections offer examples of the positions of ZFP57 binding site, ZFBS-morph-overlaps, and density-peaks in the context of known ICRs or candidate DMRs in bovine DNA. Additionally, we cover an example of how our strategy could be applied to discover novel candidate ICRs and imprinted genes.

\section{Within the Bos Taurus chromosome 29, density-plots located the ICR of the H19-IGF2 imprinted domain}

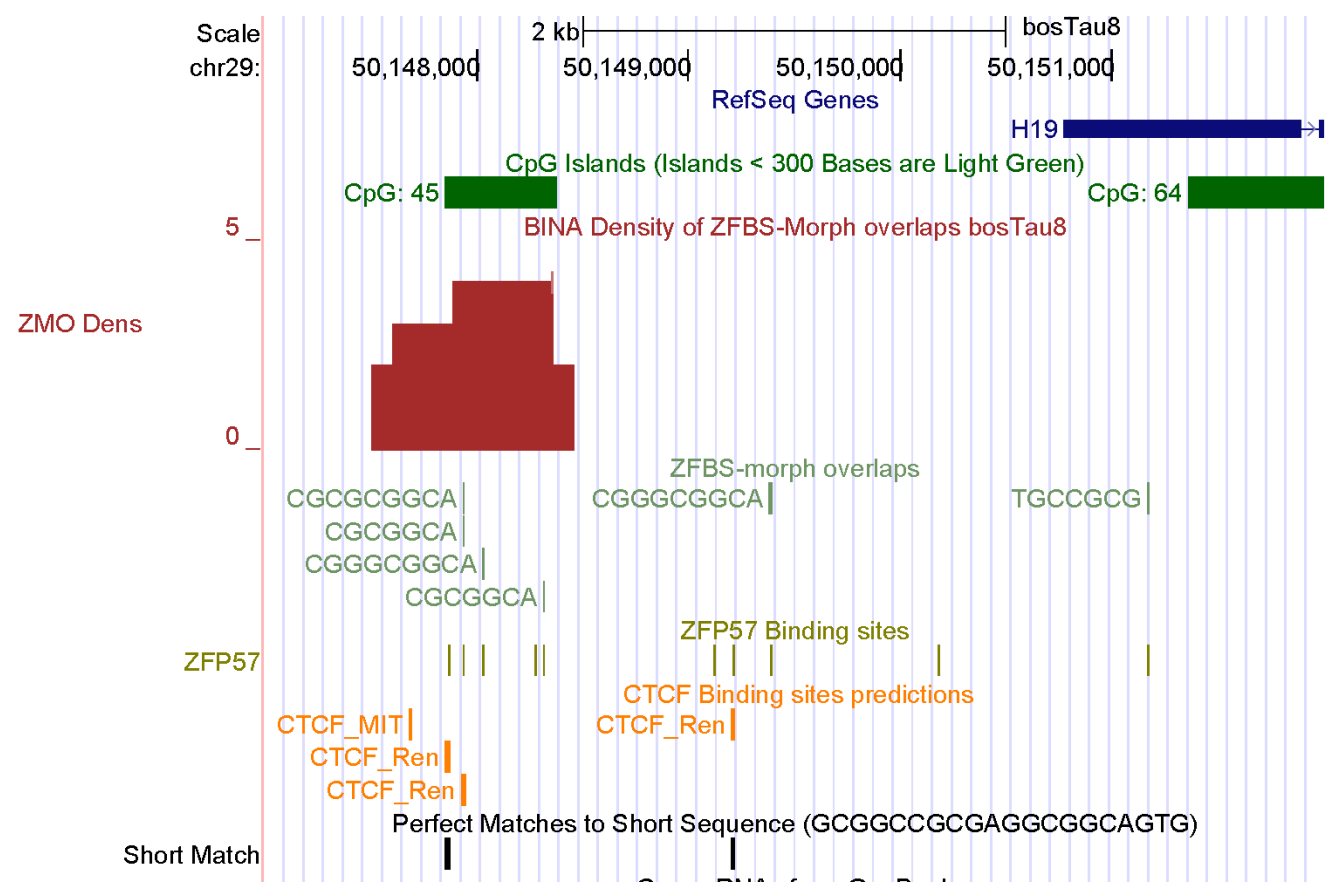

Figure 1. A robust-peak identifying the ICR of the $H 19$ - IGF2 imprinted domain in the cattle genome. From top to bottom, tracks display the positions of: cattle RefSeq genes, in pack format; the CpG islands; peaks in density-plot, in full format; ZFBS-morph overlaps, in pack format; ZFP57 binding sites, in dense format. Orange bars mark predicted CTCF binding sites. Short match corresponds to a previously reported consensus CTCF binding sequence. Note: in that plot, the peak is within a previously localized putative DMR encompassing a predicted CTCF-binding sequence. 
In mice, the expression of $H 19$, Igf2, and Ins2 is regulated by a single ICR/gDMR positioned upstream of $H 19$ [12]. H19 specifies a noncoding RNA gene transcribed from the maternal allele. Igf2, and Ins2 are expressed from the paternal allele and impact fetal growth and body size. As in mice, in cattle the H19IGF2 imprinted domain is important to normal growth and fetal development [4, 9, 28, 29]. In cloning studies, deceased newborn calves displayed abnormal expression of H19 and IGF2. In normal surviving adults, the expression of IGF2 in muscle was highly variable [30]. Furthermore, aberrant methylation of a DMR upstream of $H 19$ produced abnormal calves and LOS pathogenesis [4, 31]. In H19 DMR, a study found $33 \mathrm{CpGs}$ in 600 bps [4]. Another study detected a 300-bp DMR at approximately $6 \mathrm{~kb}$ upstream of the H19 promotor [32]. This DMR mapped to a predicted CpG island with a CTCF-binding site corresponding to consensus sequence 5' -GCGGCCGCGAGGCGGCAGTG- 3' [32].

In the density-plot of Chr29, we noticed a robust peak in a CpG island (CpG45) upstream H19 (Fig. 1). Peak-position agrees with the reported DMR in cattle DNA [32]. Furthermore, the length of CpG45 is about the same as the CpG-rich DNA (600 bps) selectively methylated in the bovine parental allele [4]. Additionally, under the density-peak, we located 3 predicted CTCF sites (Fig. 1). In our studies, we found predicted CTCF sites using a server at the University of Tennessee Health Science Center [33]. We chose that server because previously it had correctly predicted CTCF binding sites in the ICR of human H19IGF2 imprinted domain [34]. The positions of predicted sites agreed with results of ChIPs reporting the association of nuclear proteins (i.e. CTCF, RAD21, and SMC3) with chromatin [35].

For studies of cattle DNA, we chose a genomic segment (chr29:50146931-50149221) encompassing the CpG island upstream of $\mathrm{H} 19$ gene in the build BosTau8 at the UCSC genome browser. We submitted the sequence of that DNA segment to the server at the University of Tennessee Health Science Center [33]. In bovine DNA, the server predicted 5 CTCF sites (Fig. 1). One site (CTCF_Ren) corresponds to the consensus sequence (GCGGCCGCGAGGCGGCAGTG) identified previously [32]. Three sites are under the density peak and map to the $\mathrm{CpG}$ island that includes the experimentally identified bovine DMR [32]. The 5th site is downstream CpG45 (Fig. 1). Thus, we deduced that our approach correctly located the ICR position in cattle $\mathrm{H} 19$ - IGF2 imprinted domain.

\section{Within the Bos Taurus chromosome 29, density plots located the ICR of the KCNQ1 imprinted domain}

As in mice [36], the KCNQ1 imprinted domain in bovine DNA is adjacent to the H19-IGF2 domain and regulated by an ICR Known as the KvDMR1 [37]. In mice, the KvDMR1 encompasses the Kcnq1ot1 promotor and regulates imprinted expression of several protein-coding genes [28, 36, 38, 39]. This intragenic ICR is selectively methylated in oocytes but not in sperm $[28,40]$. Thus, while Kcnq1ot1 is transcribed from the paternal allele producing a noncoding RNA, the expression of several genes is repressed in the maternal allele [28]. In mice, targeted deletion of the KvDMR1 caused loss of imprinting 
and growth deficiency [41]. In cattle, defects in the KvDMR are the most common genomic region affected in LOS pathogenesis $[2,5,31,37,42]$.

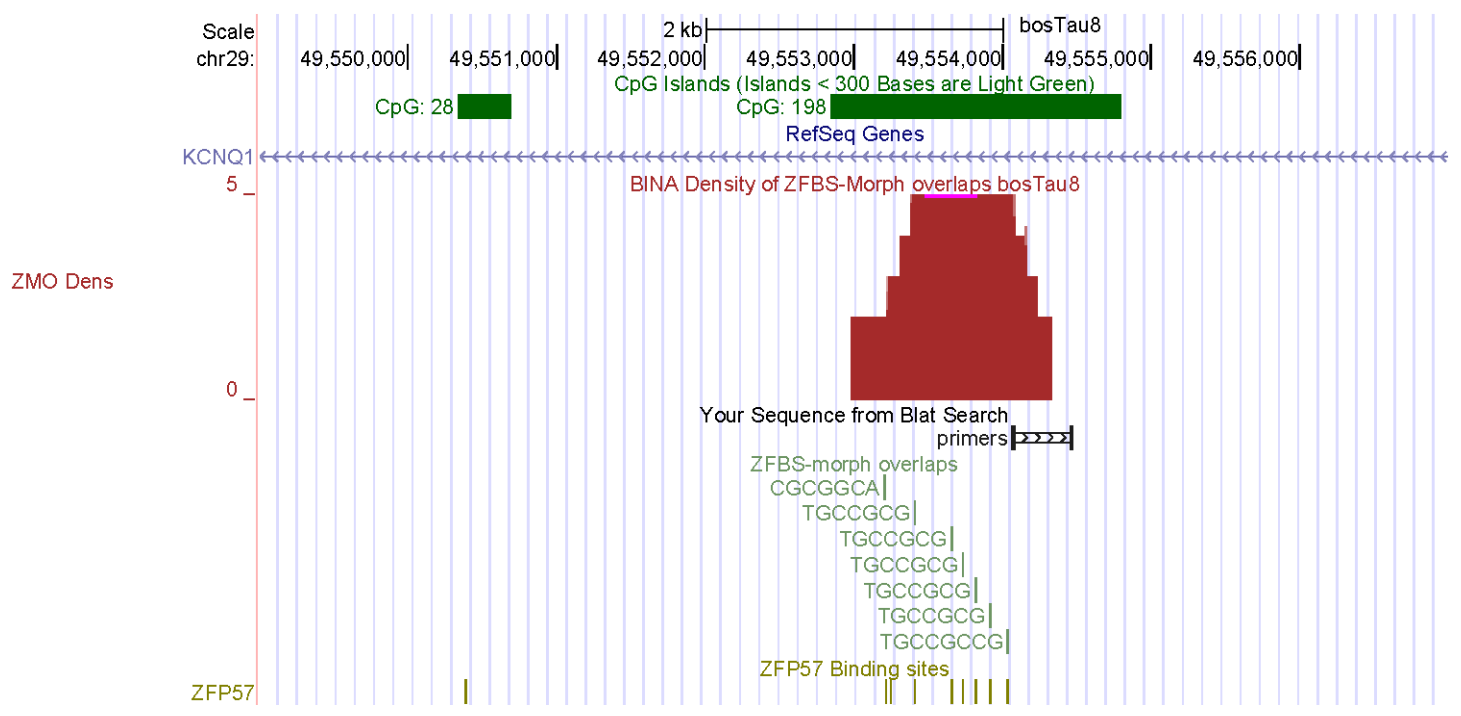

Figure 2. A robust peak pinpointing the KvDMR1 in the cattle genome. Previously, a DNA segment was amplified for locating the KvDMR1 in bovids. The plot shows the position of the two primers used in amplification reaction. These primers are in a $\mathrm{CpG}$ island that encompasses the robust peak locating the KvDMR1 in cattle DNA. ZFBS-morph overlaps are shown in packed and ZFP57 binding sites in dense formats.

In the density-plots of Bos Taurus DNA, we observed a very robust peak in one of the KCNQ1 introns (Fig. 2). In order to ensure that this peak is locating the KVDMR1, initially we selected the $5^{\prime}$ and $3^{\prime}$ ends of human KCNQ1OT1 to perform a BLAT search on the UCSC genome browser. Due to length constraints imposed by BLAT, we could not use the entire human KCNQ1OT1 as query. Nonetheless, from the BLAT output we could deduce that the peak was in the vicinity of KCNQ1OT1. For additional validation, we examined results of a study that localized cattle KvDMR1 using 2 primers to amplify an intragenic DNA. In that study, statistical analysis showed a significant difference in the methylation level between the two parental alleles -confirming that the amplified DNA corresponded to the KvDMR1 [43]. Therefore, we performed another BLAT search using the sequences of the 2 primers selected for amplifying an intragenic DNA [43]. A close-up view shows that the primers are within a relatively long CpG island that encompasses the robust peak in the density-plots (Fig. 2). Thus, that peak located the central position of the KvDMR in cattle DNA. Notably, while the KvDMR1 in mice encompasses 2 ZFBS-morph overlaps 
[19], that in cow encompasses 7. Therefore, it seems that ICRs display species-specific differences in the number of ZFBS-morph overlaps they encompass.

Within the Bos Taurus chromosome 9, density-plots revealed a candidate gDMR/ICRs within the PLAGL1 locus

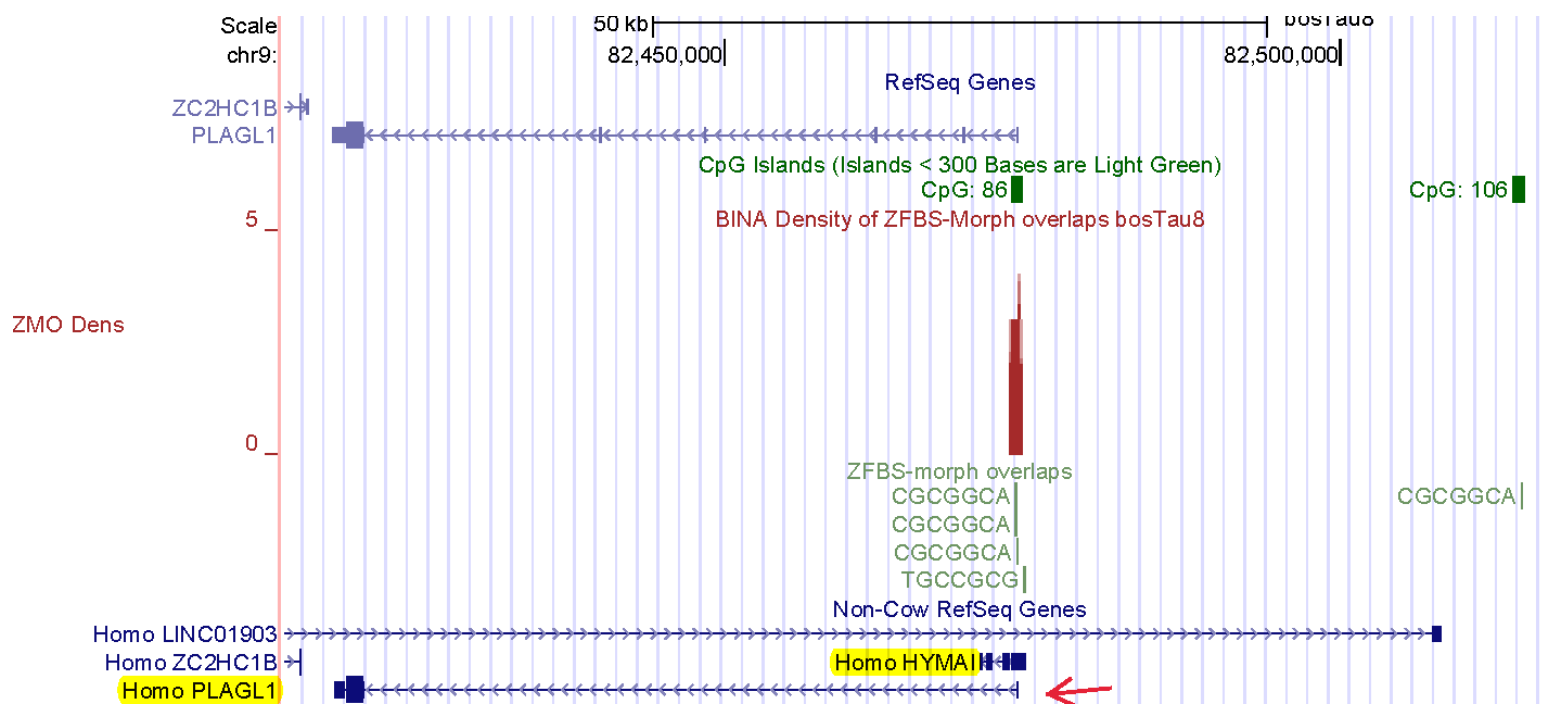

Figure 3. A robust peak predicting the ICR for imprinted expression of the PLAGL1 transcript known as $Z A C 1$. The predicted ICR is at the correct position with respect to two human imprinted transcripts: one transcript corresponds to HYMAl; the other to ZAC1.

PLAGL1 belongs to a family of zinc finger transcription factors that include PLAG1 and PLAGL2 -reviewed in [44]. One of the PLAGL1 transcripts (ZAC1) encodes a protein that inhibits tumor-cell-proliferation through the induction of cell cycle arrest and apoptosis [44]. In contrast, PLAG1 and PLAGL2 are protooncogenes. Furthermore, ZAC1 is an intragenic maternally imprinted gene. In both mouse and human genomes, transcription of ZAC1 originates within an intronic sequence in PLAGL1. That intronic region includes another imprinted gene known as $\operatorname{HYMAI}[19,45,46]$. Both ZAC1 and HYMAI are selectively expressed from the paternal allele. Their ICR/gDMR corresponds to an intragenic CpG island $[19,45,46]$. When the gDMR from human DNA was transferred into mice, this gDMR acted as an ICR and regulated allele-specific expression [47]. In LOS induced by assisted reproduction, imprinting was compromised producing biallelic expression $[2,6]$. 
As previously observed in mice [48], the cattle PLAGL1 locus encompasses a cluster of several ZFBS-Morph overlaps. In the density-plots, that cluster defines a peak (Fig. 3). However, instead of several PLAGL1 transcripts, cattle RefSeq Genes displayed only one. We suspected that this transcript corresponded to a previously reported annotation. Specifically, in a panel of maternally imprinted genes, PLAGL1 was among the loci that acquired methylation marks in an oocyte size-specific manner [49]. In that panel, a putative DMR was localized in a CPG island at the 5' end of a single transcript referred to as PLAGL1/ZAC1 [49]. To obtain clues about a complete annotation, we inspected our predicted ICR-position with respect to NonCow RefSeq Genes. In the context of human RefSeq Genes, we noticed 2 PLAGL1 transcripts and a noncoding RNA gene marked as HYMAl (Fig. 3). The 5' end of human HYMAl is in the vicinity of an imprinted $\mathrm{CpG}$ island [45]. Furthermore, as ZAC1, HYMAI is expressed from the paternal allele. Therefore: it seems likely that ZAC1 corresponds to the transcript annotated as PLAGL1 in cattle RefSeq Genes. Specifically, the genomic organization of mouse Zac1 is essentially the same as human ZAC. Both transcripts originate in a CpG island [46]. In human DNA, HYMAI TSS is intragenic [19, 45, 46]. When examined with respect to human RefSeq Genes, the density-peak also is intragenic. Furthermore, in bovine DNA the peak is in a CpG island (Fig. 3). Therefore, we are tempted to conclude that our strategy has pinpointed the reported putative ZAC1 DMR [49], and to deduce that in bovine PLAGL1 locus, the putative ZAC1 DMR is a bona fide ICR.

\section{Within Bos Taurus chromosome 9, density-plots located the ICR for the IGF2R-AIRN imprinted domain}

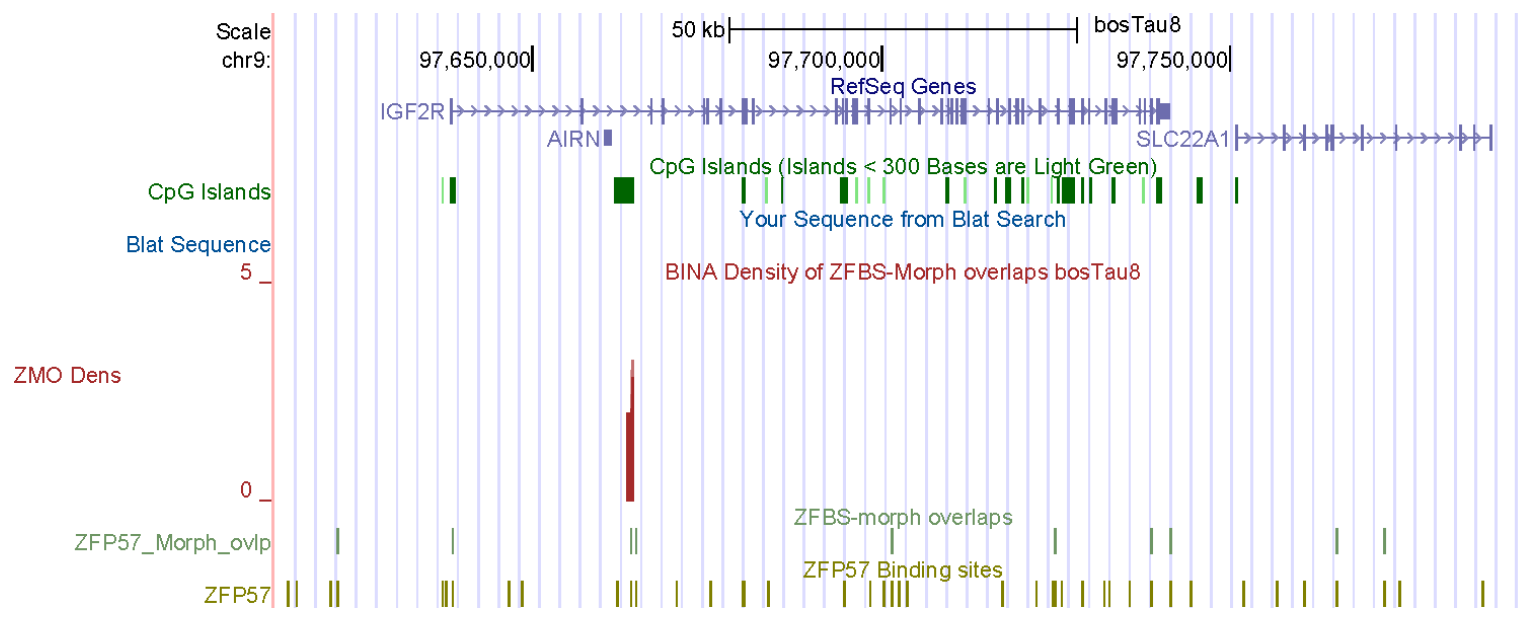

Figure 4. A robust peak defining the ICR of IGF2R imprinted domain in cattle genome. As observed for mice, in cattle the ICR in intragenic and maps to the $5^{\prime}$ end of $A I R N$. 
IGF2R impacts fetal growth and development. IGF2R also plays key roles in cell differentiation and apoptosis. Its functions include transport of IGF2 into cells and to lysosomes for degradation [50]. In mice, Igf2r knockout caused fetal overgrowth and neonatal lethality [51]. The mouse Igf2r-Airn imprinted domain includes two differentially methylated CpG islands [52]. DMR1 encompasses the Igf2r promotor. The $\mathrm{CpG}$ island that encompasses DMR2 is intragenic. In this island is incorporated the promotor of a noncoding RNA gene known as Air or Airn [53]. DMR2 regulates imprinted expression of Igf2 $r$ from the maternal and Airn from the paternal allele [54]. In Mice, deletion of DMR2 caused biallelic Igf2r expression [53]. In cattle, The IGF2R-AIRN imprinted domain also includes the DMR2 that regulates imprinted expression. As in mice, DMR2 in cattle is in the second IGF2R intron and thus intragenic [4]. As expected, bovine DMR2 is not methylated in sperm and hypermethylated in oocytes [4]. In SCNT experiments, IGF2R expression was consistently biallelic -regardless of the source of bovine embryos [4]. Bovine DMR2 is about 2 to $2.7 \mathrm{~Kb}$ and located in the second IGF2R intron [4].

While the IGF2R-AIRN imprinted domain in Bos Taurus DNA encompasses many CpG islands, one could assume that DMR1 corresponds to the island at the 5' end of IGF2R and DMR2 to the island that maps to the AIRN promotor (Fig. 4). In that context, in the density-plots, clearly apparent is a robust peak in the CPG island at the AIRN promotor (Fig. 4). Since this peak is in DMR2, we deduced that our approach pinpointed the ICR in the IGF2R-AIRN imprinted domain in bovine DNA. Notably, while the gDMR in the mouse genome encompasses 7 ZFBS-Morph overlaps [19], the gDMR in Bos Taurus DNA encompasses 3. Thus, as mentioned above, the number of ZFBS-Morph overlaps in ICRs could be species-specific.

\section{Within Bos Taurus chromosome 13, density-plots revealed a candidate for the essential ICR of the complex GNAS locus}

In both the human and mouse genomes, The GNAS locus encompasses multiple DMRs, promotors, and allele-specific transcripts [55]. Since several transcriptional variants are produced from differential exon utilization, they are collectively referred to as GNAS. The well-studied Gnas locus in mice includes three groups of protein-coding transcripts (Nesp55, XLas, and Gas). Although these transcripts share alternative exons, they are regulated by separate promotors $[55,56]$. Among the transcripts: Nesp 55 is expressed from the maternal allele; XLas from the paternal allele; Gas from both alleles [56]. Nesp55 specifies a neuroendocrine secretory protein (known as SCG6). XLas and Gas transcripts are related. Their products function in signal transmission, by G coupled hormone-receptors (GPCRs), and display distinguishable properties [57-60]. The locus also includes an imprinted gene (Nespas) transcribed into a noncoding antisense RNA [55].

At the genome browser, we noticed that in contrast to complete annotations reported for mice and humans, cattle RefSeq genes displayed only two transcripts (Fig. 5). From a publication [61], we inferred that the shorter transcript was likely to encode the Gas subunit of GPCRs. In cattle, SNPs within that transcript were associated with performance traits [61]. Even though not displayed on the browser, for 
cattle a previous study identified GNASXL as a paternally expressed transcriptional isoform. The study also described a maternally expressed transcript designated as GNAS or NESP55 [62].

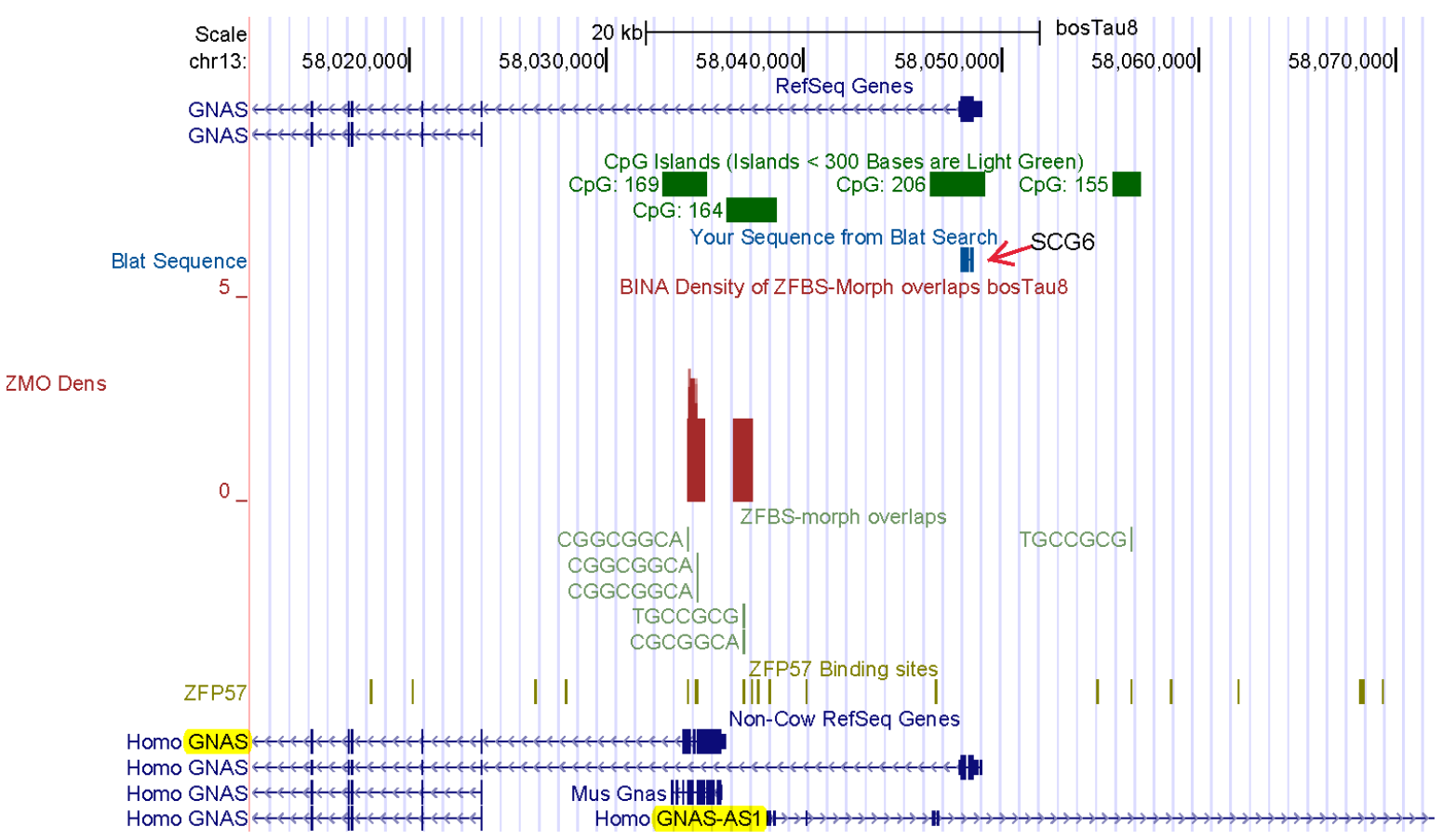

Figure 5. Locating the essential ICR in the GNAS locus. The output of a BLAT search identified the longer GNAS transcript as NESP55 (encoding SCG6). As observed for mice, two peaks define the likely position of the essential ICR of the GNAS locus in cattle DNA. The predicted ICR position seems correct with respect to the annotation of Non-Cow RefSeq Genes including human GNAS-AS1.

Because the UCSC genome browser's gene annotations appeared incomplete, we performed a BLAT search to locate NESP55 in Bos Taurus DNA. For query we chose the amino acid sequence of human SCG6. Based on the output, we deduced that on the browser, the longer GNAS transcript corresponded to NESP55 (Fig. 5). Cattle NESP55 transcripts are expressed monoallelically in many tissues [62]. Furthermore, in cattle, the GNAS locus includes a DMR (a putative ICR) that is hypomethylated in the paternal allele and hypermethylated in the maternal allele [62].

In the density-plots, we observed two clusters of ZFBS-Morph overlaps producing two peaks (Fig. 5). Similarly, a previous study also noticed two clusters of ZFBS-Morph overlaps in the mouse locus [19]. In cattle DNA, both peaks are in the $1^{\text {st }}$ intron of the transcript corresponding to NESP55 (Fig. 5). Notably, the essential ICR in human locus includes two DMRs: one DMR maps to the first exon of XLas; the other is 
near GNAS-AS1 TSS [55]. With respect to human RefSeq Genes, peak positions in cattle DNA map to human XLas and to a region upstream GNAS-AS1 (Fig. 5). In toto, we could infer that our strategy predicted the genomic position of the essential ICR in the complex GNAS locus in the Bos Taurus DNA.

\section{Within the Bos Taurus chromosome 18, density-plots located a candidate ICR for the PEG3 imprinted domain}

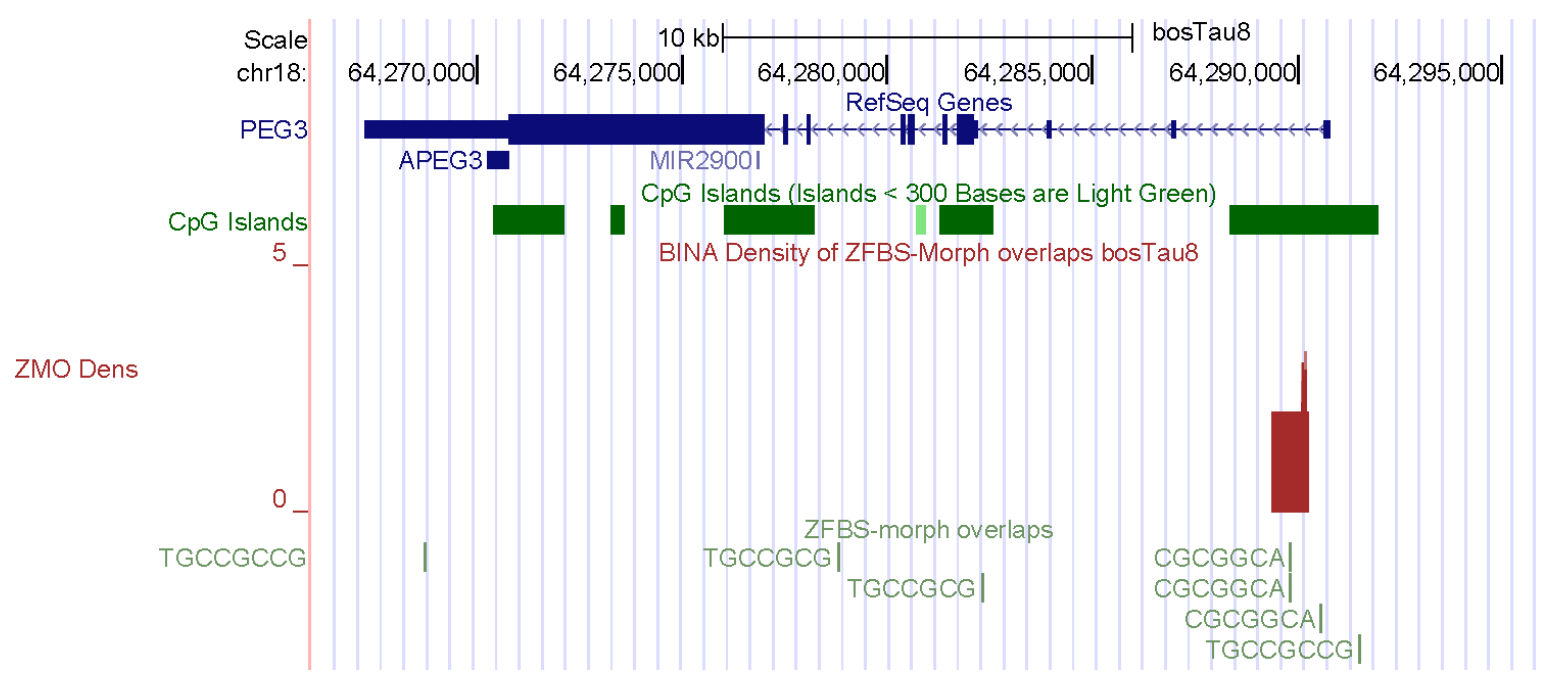

Figure 6. A robust peak locating the ICR of PEG3 imprinted domain. As detailed in the text, this peak is within the $\mathrm{CpG}$ island that is methylated in a parent-of-origin-specific manner.

In mice, the Peg3 imprinted domain encompasses several genes [63]. Peg3 encodes a relatively large nuclear protein with 12 zinc fingers of $\mathrm{C} 2 \mathrm{H} 2$ type [64]. This protein plays a key role in reactions of female mice to her litters [65]. A mutation in Peg3 caused a striking impairment of maternal behavior. Due to the dearth of maternal care, litters developed poorly and often died [65]. Mechanistically, mutant mothers were deficient in milk ejection -partly due to defective neuronal connectivity, as well as reduced oxytocin neurons in the hypothalamus [65]. In domesticated animals, oxytocin is an indicator of psychological and social well-being [66]. As observed in mice and humans, PEG3 is expressed from the paternal allele in cattle $[9,67]$. In cattle, the PEG3 domain underwent a global loss of imprinting in fetal overgrowth syndrome induced by assisted reproduction [2].

Sequence analyses have predicted that in Bos Taurus, the PEG3 imprinted domain encompasses many $\mathrm{CpG}$ islands [67]. Consistent with this prediction, at the genome browser we observed several $\mathrm{CpG}$ islands in bovine DNA (Fig. 6). In cattle, the island encompassing the PEG3 promotor was the only area that 
showed DMR status [67]. Notably, in the density-plots we observed a peak in that DMR (Fig. 6). Since the DMR was methylated in an allele-specific manner [67], we could deduce that the peak pinpointed the ICR in cattle PEG3 imprinted domain. Furthermore, earlier studies of mice revealed that clusters of ZFBSmorph overlaps mapped to functionally important landmarks -including DNase I hypersensitive sites and repressive H3K9me3 marks [19]. In mouse, these clusters were in the $1^{\text {st }}$ Peg3 intron. Likewise, for cattle we observe an intragenic peak. Therefore, our strategy pinpointed the central portion of the ICR in cattle PEG3 imprinted domain (Fig. 6).

\section{Within Bos Taurus chromosome 4, density-plots located the intragenic ICR regulating allele-specific expression of MEST isoform}

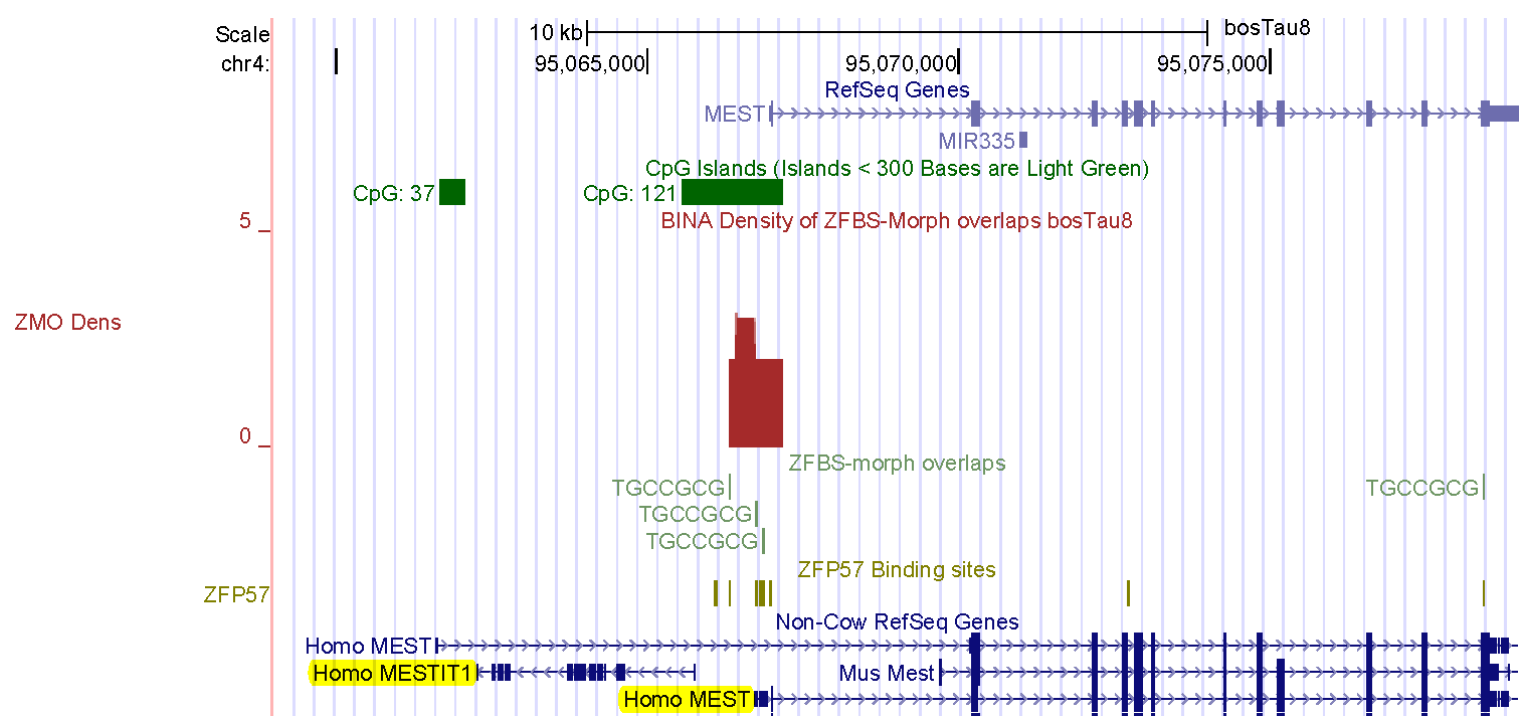

Figure 7. A robust-density-peak locating the ICR for imprinted expression of the MEST variant in cattle DNA. This location is presented in the context of Non-Cow RefSeq Genes for human MESTIT1 and MEST transcriptional variant.

MEST is a member of the $\alpha / \beta$-hydrolase fold family of enzymes [68]. In mice, the TSS of a paternally expressed transcript originates from the $1^{\text {st }}$ Mest intron $[68,69]$. This transcript is also known as Peg1. An intragenic ICR regulates its expression selectively from the paternal allele. In cattle, among 8 investigated genes, only MEST showed differential expression in day 21 parthenogenetic embryos [70]. In mice, the product of Peg1 impacts maternal behavior [71]. This conclusion was reached from studies of Peg1 deficient females. Even though these females displayed a normal investigative behavior, they lacked appropriate maternal response [71]. Consequently, their newborn litters had a low survival rate 
[71]. Furthermore, Peg1 deficient females displayed abnormal maternal activities including impaired placentophagia.

In bovine DNA, methylation imprints are established in an oocyte size-specific manner [49]. In a panel of maternally imprinted genes, putative DMRs were localized in several imprinted loci including MEST. CpG analyses deduced that the putative Peg1/MEST DMR maps to a CpG island [49]. Consistent with this prediction, density-plots revealed a peak in a CpG island in the MEST locus (Fig. 7). Since on the browser we observed a single MEST transcript, we inspected peak-position with respect to Non-Cow RefSeq Genes. In the context of human RefSeq Genes, the peak is upstream of MESTIT1 and near one of the MEST short isoforms. As observed for MEST, MESTIT1 is expressed from the paternal allele [72]. Thus, the density-plots correctly pinpointed the ICR regulating the expression of PEG1/MEST transcript in bovine DNA. Furthermore, the plots demonstrated the correspondence of this predicted ICR to the reported imprinted DMR in cattle DNA [49].

\section{Within Bos Taurus chromosome 13, an intragenic peak located the ICR regulating allele-specific expression of NNAT}

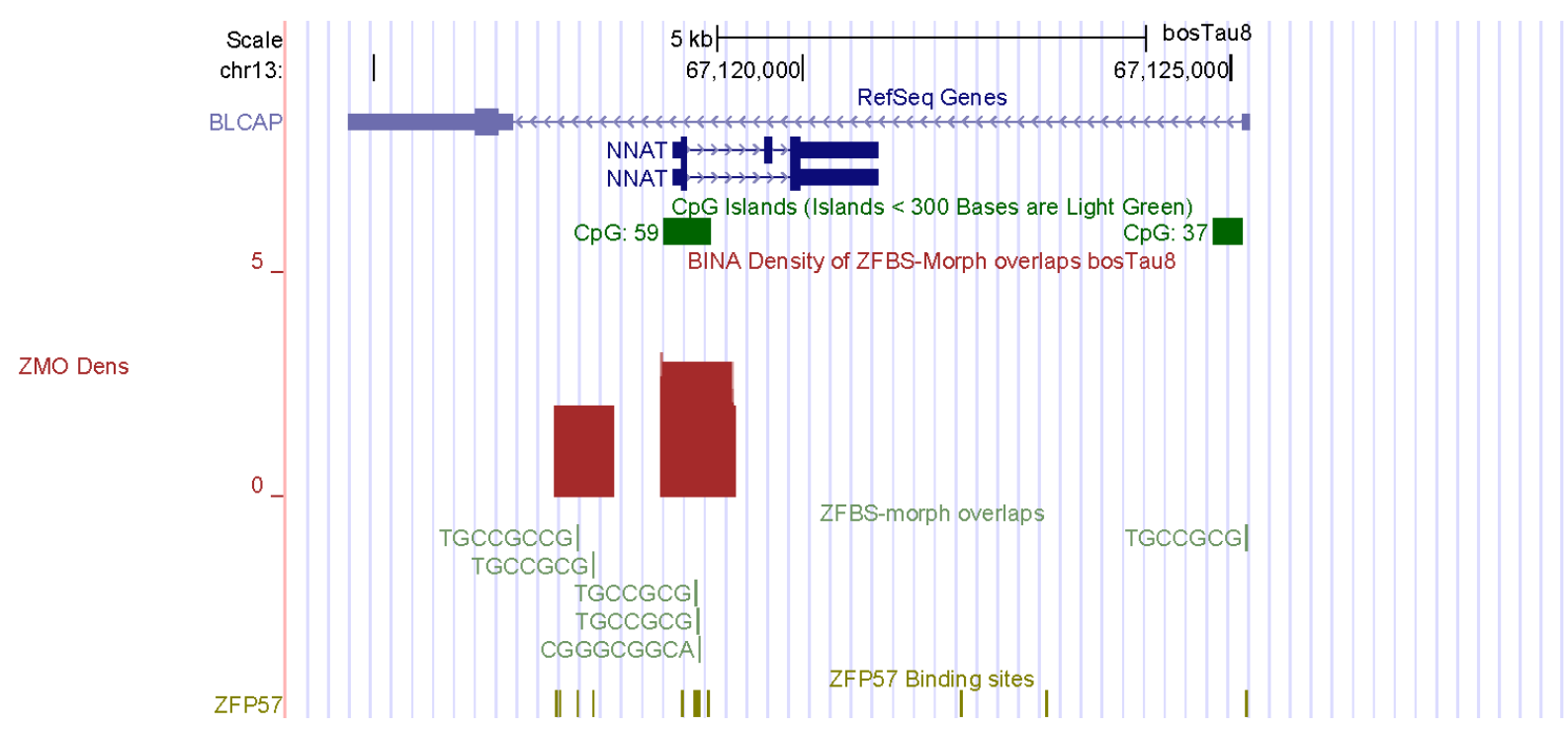

Figure 8. A robust-density-peak locating a candidate ICR for imprinted NNAT expression in cattle DNA. This peak is in CpG59 and maps to a known cattle DMR.

The neuronatin gene (NNAT) is expressed during postnatal brain development [ [73]. In mice, Nnat is located within the single intron of Blcap [74]. Also known as Peg5, Nnat is an imprinted gene. Blcap is expressed biallelically [74]. From Nnat are produced several alternatively spliced isoforms [74]. In human, 
the CpG island in NNAT promotor was differentially methylated in all examined tissues [75]. Therefore, that island corresponds to the ICR that regulate NNAT expression [75].

As in humans and mice [74-76], in cattle two NNAT transcripts are expressed from the paternal allele [77]. In plots of cattle DNA, we noticed a CpG island near the BLCAP TSS. Another island (CpG59) is intragenic (Fig. 8). In the density-plots, we did not find a peak in the island near BLCAP, a finding that is consistent with $B L C A P$ biallelic expression. In BLCAP intron, we noticed 2 peaks. One of the peaks is more robust. It is located in CpG59 and encompasses both NNAT TSS and promotor (Fig. 8). This peak is the likely position of the ICR that regulates imprinted NNAT expression in bovine DNA (Fig. 8). Overall, robustness of peaks depends on the number of ZFBS-morph overlaps they encompass. Peaks that cover 2 ZFBS-morph overlaps could be true or false positive [26]. Peaks that cover 3 are more robust.

\section{Within Bos Taurus chromosome 21, a peak predicted a candidate ICR for regulating expression of MEG8}

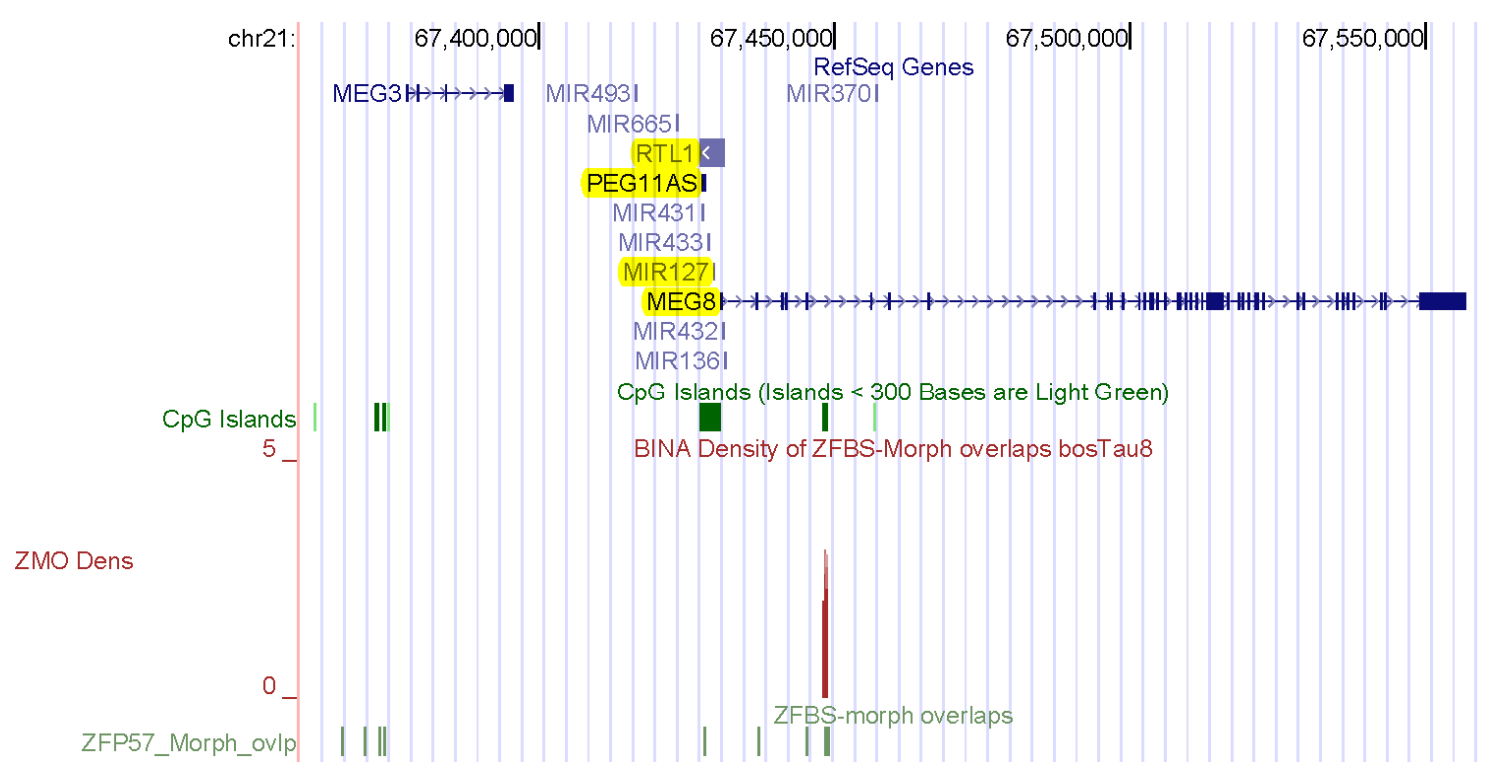

Figure 9. A robust peak predicting the ICR for imprinted expression of MEG8. This peak is intragenic and maps to a CpG island.

In cattle, the DLK1-DIO3 imprinted cluster includes a maternally-expressed gene (MEG8) transcribed into a noncoding RNA [78]. In an 8-wk-old animal, MEG8 was preferentially expressed in skeletal muscle [78]. In Angus calves, maternal diet during pregnancy impacted MEG8 expression in longissimus dorsi muscle [79]. In adult cattle, MEG8 was expressed in several tissues -including heart, liver, spleen, lung, kidney, brain, subcutaneous fat and skeletal muscle [80]. In heterozygous cattle, MEG8 was expressed 
from only one of the two parental alleles. In the density-plots, we observed a robust peak predicting a candidate ICR for allele-specific expression of cattle MEG8. This peak is intragenic and maps to a CpG island (Fig. 9).

\section{Within Bos Taurus chromosome 26, a peak predicted a candidate ICR for imprinted INPP5F_V2 expression}

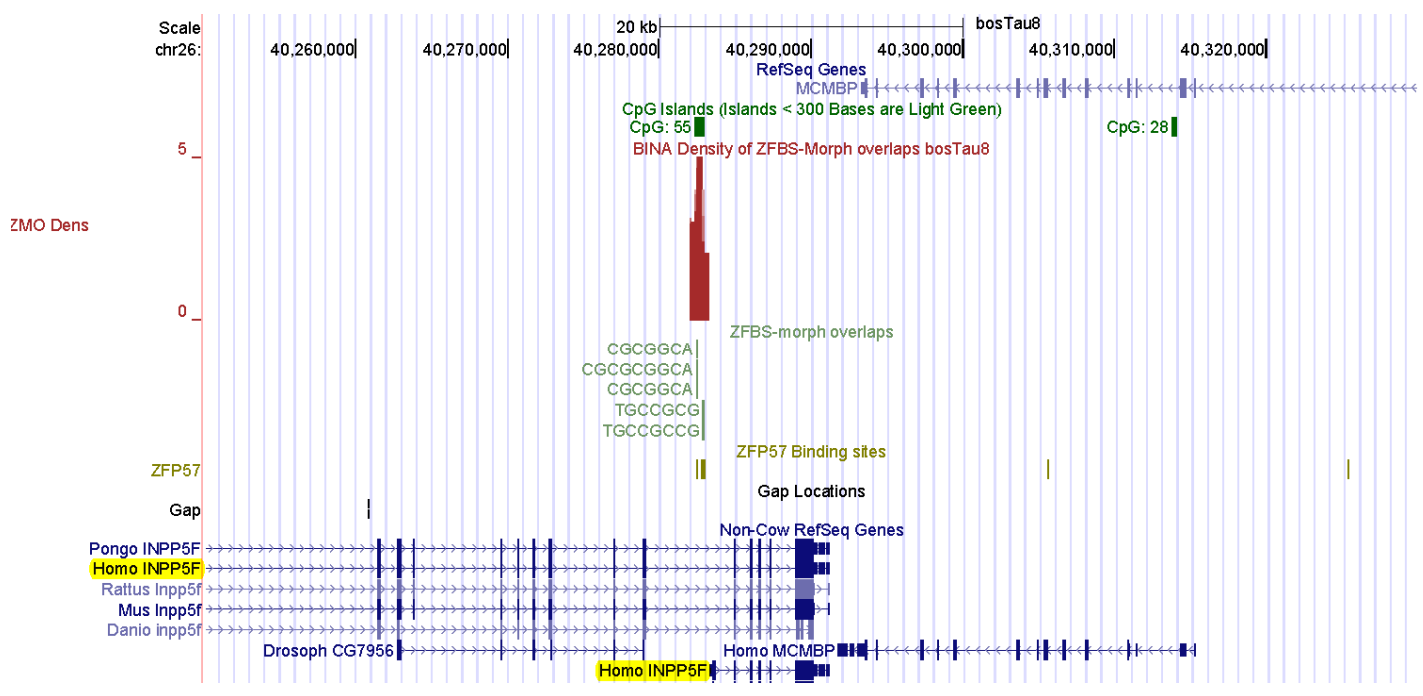

Figure 10. With respect to Non-Cow RefSeq Genes, a robust-peak predicted the ICR of INPP5F_V2 in cattle DNA. As observed for mice and humans, this peak is in an intragenic $\mathrm{CpG}$ island.

INPP5F is an inositol 4-phosphatase that functions in the endocytic pathway [81]. In mice, Inpp5f_v2 was identified as an imprinted gene in the brain [82]. Inpp5f_v2 is a variant of Inpp5f. It is a retrogene with a unique alternative first exon. Inpp5f_v2 transcription originates in one of the Inpp5f introns. While Inpp5f is biallelically expressed, Inpp5f_v2 is transcribed from the paternal allele [82]. Furthermore, mouse Inpp5f and human INPP5F share sequence similarity. In mouse Inpp5f locus, CpG analyses identified two CpG islands [82]. CpG1 is near the 5' end of Inpp5f, CpG2 is at 5' end of Inpp5f_v2. Bisulfite sequencing of the CpG1 island showed that both alleles were hypomethylated, as would be expected for a nonimprinted transcriptionally active gene. In the brain, $\mathrm{CpG} 2$ was methylated on the maternal allele, but not on the paternal allele. Thus, the intragenic island regulates imprinted Inpp5f_v2 expression [82]. Similarly, the promotor of human INPPF5_V2 transcript is embedded within a maternally methylated DMR [83].

Literature surveys we could not find reports concerning cattle INPPF5_V2. Furthermore, the genome browser did not include any annotation for the INPPF5 locus in cattle DNA. The only annotated gene 
corresponded to MCMBP (Fig. 10). However, we were able to locate INPP5F transcripts in the context of Non-Cow RefSeq Genes. With respect to human genes, INPPF5 is upstream of MCMBP -as observed in human and mouse genomic DNA. Since we were in correct genomic area in cattle DNA, we could ask whether the density-plots included a candidate ICR for allele-specific expression. In plots, we noticed a robust peak in a $\mathrm{CpG}$ island (CpG55) in cattle DNA. With respect to human transcripts, this peak maps to the 5' end of INPPF5_V2. Therefore, CpG55 is the likely ICR position in INPPF5 locus in cattle DNA. As first described for mice, this ICR is intragenic [82]. Thus, our strategy predicted a candidate ICR for imprinted bovine INPPF5_V2 expression from bovine DNA (Fig. 10).

\section{Density-plots could facilitate locating candidate ICRs in bovine DNA}

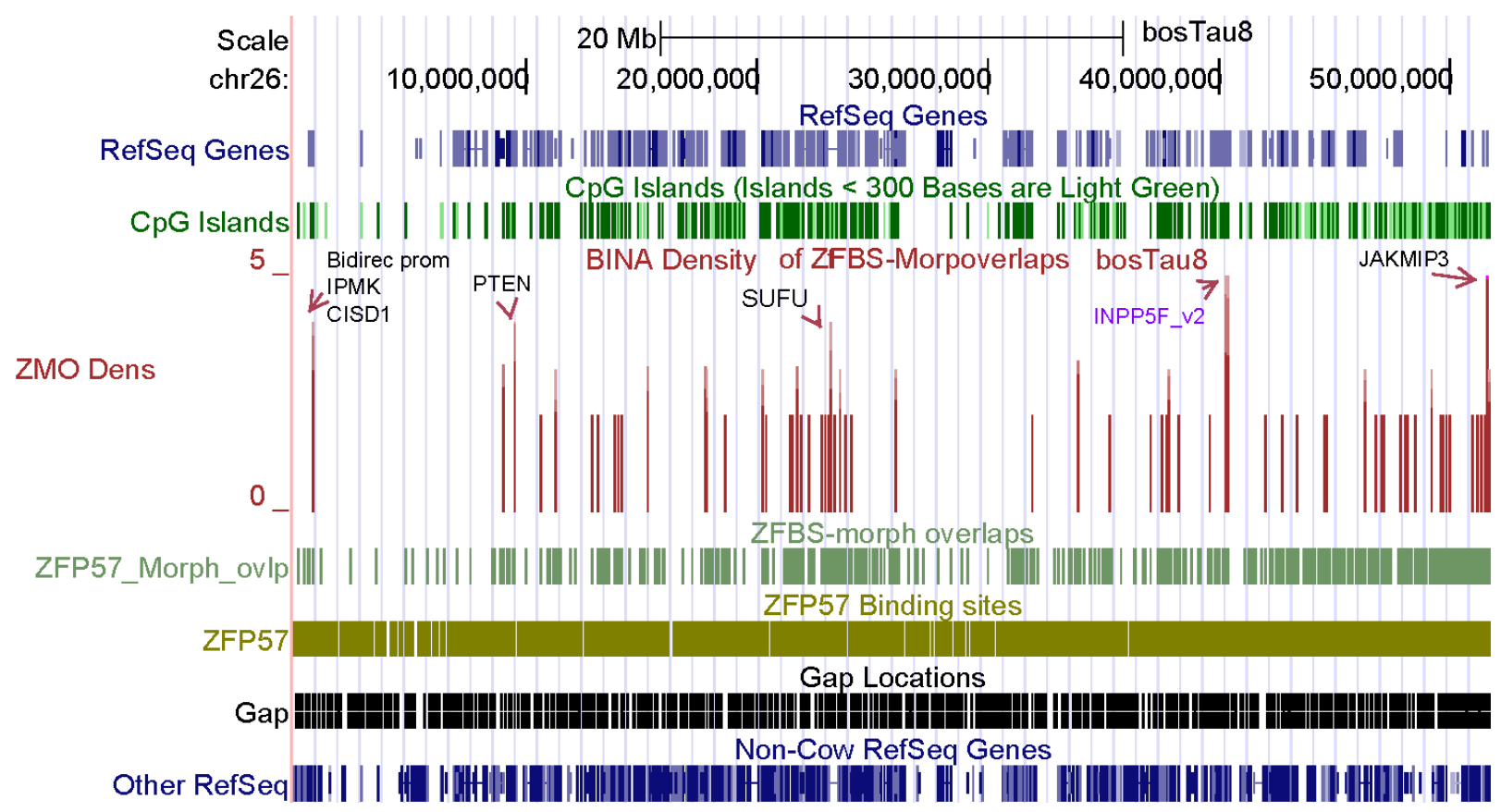

Figure 11. A snapshot of the density-plot obtained for the entire Chr26. Peaks covering 2 ZFBS-morph could be true or false-positives. Robust peaks cover 3 or more overlaps. Purple indicates the position of candidate ICR for imprinted INPPF5_V2 expression. Additional robust peaks correspond to predicted ICRs. Arrows mark representative examples of such ICRs with respect to nearby genes.

Since the density-plots were created genome-wide, we could view peak positions along the DNA in an entire chromosome. For example, examine a snapshot from the UCSC genome browser presenting peak positions along the entire DNA in Chr26 (Fig. 11). In the build bosTau8, this chromosome covers greater 
than 51,7 Mb DNA. We find that even along an entire chromosome, many peaks are almost fully resolved. This plot demonstrates clearly that density peaks occur infrequently in cattle DNA. This finding is expected since CpG frequency is relatively low in animal DNA [84]. Since the peaks encompass several CpGs, they represent uncommon events along genomic DNA. Additionally, as one would expect, peaks covering 3 or more ZFBS-morph overlaps are sparser than those that covering 2 (Fig. 11). Since in plots, several peaks have correctly identified regions that include known ICRs and DMRs (Figs. 1-10), we propose that additional peaks correspond to candidate ICRs. Examples include a robust peak in a bidirectional promotor regulating IPMK and CISD1, a peak associated with PTEN, and two intragenic peaks regulating SUFU, and JAKMIP3 expression (Fig. 11).

To facilitate data interpretation, we have tailored our datasets for upload onto the UCSC the genome browser to create custom tracks. The browser offers a great resource for studies of genomic DNA sequences in higher organisms [85-87]. On the browser, default tracks facilitate viewing our datasets in the context of genomic landmarks including genes, transcripts, the CpG islands, SNPs and much more [88]. At the Purdue University Research Repository (PURR), you can access and download the datasets listed below:

-The positions of ZFBS and ZFBS-Morph overlaps in the build bosTau 8 of the cow genome https://purr.purdue.edu/publications/3359/1

-Density of ZFBS-Morph overlaps in the build bosTau8 of the cow genome

https://purr.purdue.edu/publications/3360/1

At PURR, you can also access and download datasets for studies of genomic imprinting in human, mouse, and dog. For the Bos Taurus genome, you can view peak positions along an entire chromosome (Fig. 11). You can even zoom into a desired region to obtain closeup views (Figs. 1-10). In closeup views, you can inspect peak positions with respect to genes, transcripts, CpG islands, RefSeq Genes, Non-Cow RefSeq Genes, and SNPs $[87,89]$. Furthermore, it is well known that most ICRs regulate allele-specific expression of nearby genes. Therefore, since the browser is user-friendly [87], with our datasets, you can easily discover candidate ICRs and imprinted genes for experimental validations. For an overview about how to use the UCSC genome browser, see $[86,88]$.

\section{METHODS}

\section{Marking the genomic positions of ZFP57 binding site and the ZFBS-morph overlaps}

At the UCSC genome browser, we selected the build bosTau8 to obtain and download the nucleotide sequences of cow chromosomes. Initially, we wrote a Perl script to determine the genomic positions of the reported hexameric ZFP57 binding site [14], and sequences of the ZFBS-morph overlaps [48]. The script opened the file containing the nucleotide sequence of a specified chromosome, as well as either 
the file containing the ZFP57 binding site or sequences of the ZFBS-morph overlaps. With UNIX subroutines, we combined the outputs obtained for various chromosomes for display at the browser.

Creating plots of the density of ZFBS-morph overlaps in genomic DNA

With a Perl script, we established the genomic positions of DNA segments that covered 2 or more closely spaced ZFBS-morph overlaps. That script opened the file containing the positions of ZFBS-morph overlaps for a specified chromosome. Subsequently, the script scanned the file to count and report the number of ZFBS-morph overlaps within a sliding window consisting of 850-bases. To remove background noise, the script ignored isolated overlaps. Next, using a subroutine we combined and tailored the outputs of the program for display as a custom track on the UCSC genome browser.

\section{REFERENCES}

1. Smith LC, Therrien J, Filion F, Bressan F, Meirelles FV: Epigenetic consequences of artificial reproductive technologies to the bovine imprinted genes SNRPN, H19/IGF2, and IGF2R. Front Genet 2015, 6:58.

2. Chen Z, Hagen DE, Elsik CG, Ji T, Morris CJ, Moon LE, Rivera RM: Characterization of global loss of imprinting in fetal overgrowth syndrome induced by assisted reproduction. Proc Natl Acad Sci U S A 2015, 112(15):4618-4623.

3. Liu JH, Yin S, Xiong B, Hou Y, Chen DY, Sun QY: Aberrant DNA methylation imprints in aborted bovine clones. Mol Reprod Dev 2008, 75(4):598-607.

4. Smith LC, Suzuki J, Jr., Goff AK, Filion F, Therrien J, Murphy BD, Kohan-Ghadr HR, Lefebvre R, Brisville AC, Buczinski $S$ et al: Developmental and epigenetic anomalies in cloned cattle. Reproduction in domestic animals = Zuchthygiene 2012, 47 Suppl 4:107-114.

5. Urrego R, Rodriguez-Osorio N, Niemann H: Epigenetic disorders and altered gene expression after use of Assisted Reproductive Technologies in domestic cattle. Epigenetics 2014, 9(6):803815.

6. O'Doherty AM, MacHugh DE, Spillane C, Magee DA: Genomic imprinting effects on complex traits in domesticated animal species. Front Genet 2015, 6:156.

7. O'Doherty AM, McGettigan P, Irwin RE, Magee DA, Gagne D, Fournier E, Al-Naib A, Sirard MA, Walsh CP, Robert $C$ et al: Intragenic sequences in the trophectoderm harbour the greatest proportion of methylation errors in day 17 bovine conceptuses generated using assisted reproductive technologies. BMC Genomics 2018, 19(1):438.

8. Magee DA, Spillane C, Berkowicz EW, Sikora KM, MacHugh DE: Imprinted loci in domestic livestock species as epigenomic targets for artificial selection of complex traits. Anim Genet 2014, 45 Suppl 1:25-39. 
9. Tian XC: Genomic imprinting in farm animals. Annu Rev Anim Biosci 2014, 2:23-40.

10. Strogantsev R, Krueger F, Yamazawa K, Shi H, Gould P, Goldman-Roberts M, McEwen K, Sun B, Pedersen R, Ferguson-Smith AC: Allele-specific binding of ZFP57 in the epigenetic regulation of imprinted and non-imprinted monoallelic expression. Genome Biol 2015, 16:112.

11. Stewart KR, Veselovska L, Kelsey G: Establishment and functions of DNA methylation in the germline. Epigenomics 2016, 8(10):1399-1413.

12. Barlow DP, Bartolomei MS: Genomic imprinting in mammals. Cold Spring Harbor perspectives in biology 2014, 6(2).

13. Bartolomei MS, Tilghman SM: Genomic imprinting in mammals. Annual review of genetics 1997, 31:493-525.

14. Quenneville S, Verde G, Corsinotti A, Kapopoulou A, Jakobsson J, Offner S, Baglivo I, Pedone PV, Grimaldi G, Riccio A et al: In embryonic stem cells, ZFP57/KAP1 recognize a methylated hexanucleotide to affect chromatin and DNA methylation of imprinting control regions. $\mathrm{Mol}$ Cell 2011, 44(3):361-372.

15. Strogantsev R, Ferguson-Smith AC: Proteins involved in establishment and maintenance of imprinted methylation marks. Brief Funct Genomics 2012, 11(3):227-239.

16. Helleboid PY, Heusel M, Duc J, Piot C, Thorball CW, Coluccio A, Pontis J, Imbeault M, Turelli P, Aebersold $\mathrm{R}$ et al: The interactome of KRAB zinc finger proteins reveals the evolutionary history of their functional diversification. EMBO J 2019, 38(18):e101220.

17. Huntley S, Baggott DM, Hamilton AT, Tran-Gyamfi M, Yang S, Kim J, Gordon L, Branscomb E, Stubbs $L$ : A comprehensive catalog of human KRAB-associated zinc finger genes: insights into the evolutionary history of a large family of transcriptional repressors. Genome Res 2006, 16(5):669-677.

18. Riso V, Cammisa M, Kukreja H, Anvar Z, Verde G, Sparago A, Acurzio B, Lad S, Lonardo E, Sankar $A$ et al: ZFP57 maintains the parent-of-origin-specific expression of the imprinted genes and differentially affects non-imprinted targets in mouse embryonic stem cells. Nucleic Acids Res 2016, 44(17):8165-8178.

19. Bina M: Imprinted control regions include composite DNA elements consisting of the ZFP57 binding site overlapping MLL1 morphemes. Genomics 2017, 109:265-273.

20. Ruthenburg AJ, Allis CD, Wysocka J: Methylation of lysine 4 on histone H3: intricacy of writing and reading a single epigenetic mark. Mol Cell 2007, 25(1):15-30.

21. Birke M, Schreiner S, Garcia-Cuellar MP, Mahr K, Titgemeyer F, Slany RK: The MT domain of the proto-oncoprotein MLL binds to CpG-containing DNA and discriminates against methylation. Nucleic Acids Res 2002, 30(4):958-965.

22. Bach C, Mueller D, Buhl S, Garcia-Cuellar MP, Slany RK: Alterations of the CxxC domain preclude oncogenic activation of mixed-lineage leukemia 2. Oncogene 2009, 28(6):815-823. 
23. Ayton PM, Chen EH, Cleary ML: Binding to nonmethylated CpG DNA is essential for target recognition, transactivation, and myeloid transformation by an MLL oncoprotein. Mol Cell Biol 2004, 24(23):10470-10478.

24. Bina M: Gene regulation. Methods Mol Biol 2013, 977:1-11.

25. Cosgrove MS, Patel A: Mixed lineage leukemia: a structure-function perspective of the MLL1 protein. FEBS J 2010, 277(8):1832-1842.

26. Bina $M$, Wyss P: Simultaneous discovery of candidate imprinted genes and Imprinting Control Regions in the mouse genome. bioRxiv 2019.

27. Bina $M$ : Discovering candidate imprinted genes and imprinting control regions in the human genome. BMC Genomics 2020, 21(1):378.

28. Verona RI, Mann MR, Bartolomei MS: Genomic imprinting: intricacies of epigenetic regulation in clusters. Annu Rev Cell Dev Biol 2003, 19:237-259.

29. Curchoe CL, Zhang S, Yang L, Page R, Tian XC: Hypomethylation trends in the intergenic region of the imprinted IGF2 and H19 genes in cloned cattle. Anim Reprod Sci 2009, 116(3-4):213-225.

30. Yang L, Chavatte-Palmer P, Kubota C, O'Neill M, Hoagland T, Renard JP, Taneja M, Yang X, Tian $X C$ : Expression of imprinted genes is aberrant in deceased newborn cloned calves and relatively normal in surviving adult clones. Mol Reprod Dev 2005, 71(4):431-438.

31. Hori N, Nagai M, Hirayama M, Hirai T, Matsuda K, Hayashi M, Tanaka T, Ozawa T, Horike S: Aberrant CpG methylation of the imprinting control region KvDMR1 detected in assisted reproductive technology-produced calves and pathogenesis of large offspring syndrome. Anim Reprod Sci 2010, 122(3-4):303-312.

32. Hansmann T, Heinzmann J, Wrenzycki C, Zechner U, Niemann H, Haaf T: Characterization of differentially methylated regions in 3 bovine imprinted genes: a model for studying human germ-cell and embryo development. Cytogenet Genome Res 2011, 132(4):239-247.

33. Ziebarth JD, Bhattacharya A, Cui Y: CTCFBSDB 2.0: a database for CTCF-binding sites and genome organization. Nucleic Acids Res 2013, 41(Database issue):D188-194.

34. Bina M: Assessment of the CTCF Binding Sites and Repeat-Positions Upstream the Human H19 Gene. bioRxiv 2018, https://www.biorxiv.org/content/10.1101/250407v1.

35. ENCODE: A user's guide to the encyclopedia of DNA elements (ENCODE). PLOS Biol 2011, 9(4):e1001046.

36. Bartolomei MS: Genomic imprinting: employing and avoiding epigenetic processes. Genes Dev 2009, 23(18):2124-2133.

37. Robbins KM, Chen Z, Wells KD, Rivera RM: Expression of KCNQ10T1, CDKN1C, H19, and PLAGL1 and the methylation patterns at the KvDMR1 and H19/IGF2 imprinting control regions is conserved between human and bovine. J Biomed Sci 2012, 19:95. 
38. Engemann S, Strodicke M, Paulsen M, Franck O, Reinhardt R, Lane N, Reik W, Walter J: Sequence and functional comparison in the Beckwith-Wiedemann region: implications for a novel imprinting centre and extended imprinting. Hum Mol Genet 2000, 9(18):2691-2706.

39. Lewis A, Green K, Dawson C, Redrup L, Huynh KD, Lee JT, Hemberger M, Reik W: Epigenetic dynamics of the Konq1 imprinted domain in the early embryo. Development 2006, 133(21):4203-4210.

40. Tycko B, Morison IM: Physiological functions of imprinted genes. Journal of cellular physiology 2002, 192(3):245-258.

41. Fitzpatrick GV, Soloway PD, Higgins MJ: Regional loss of imprinting and growth deficiency in mice with a targeted deletion of KvDMR1. Nat Genet 2002, 32(3):426-431.

42. Li Y, Donnelly CG, Rivera RM: Overgrowth Syndrome. Vet Clin North Am Food Anim Pract 2019, 35(2):265-276.

43. Wang M, Li D, Zhang M, Yang W, Cui Y, Li S: Methylation of KvDMR1 involved in regulating the imprinting of CDKN1C gene in cattle. Anim Genet 2015, 46(4):354-360.

44. Juma AR, Damdimopoulou PE, Grommen SV, Van de Ven WJ, De Groef B: Emerging role of PLAG1 as a regulator of growth and reproduction. The Journal of endocrinology 2016, 228(2):R45-56.

45. Arima T, Drewell RA, Oshimura M, Wake N, Surani MA: A novel imprinted gene, HYMAl, is located within an imprinted domain on human chromosome 6 containing ZAC. Genomics 2000, 67(3):248-255.

46. Smith RJ, Arnaud P, Konfortova G, Dean WL, Beechey CV, Kelsey G: The mouse Zac1 locus: basis for imprinting and comparison with human ZAC. Gene 2002, 292(1-2):101-112.

47. Arima T, Yamasaki K, John RM, Kato K, Sakumi K, Nakabeppu Y, Wake N, Kono T: The human HYMAI/PLAGL1 differentially methylated region acts as an imprint control region in mice. Genomics 2006, 88(5):650-658.

48. Bina $M$, Wyss $P$, Song XC: Datasets on the genomic positions of the MLL1 morphemes, the ZFP57 binding site, and ZFBS-Morph overlaps in the build $\mathrm{mm} 9$ of the mouse genome. Data Brief 2017, 13:202-207.

49. O'Doherty AM, O'Shea LC, Fair T: Bovine DNA methylation imprints are established in an oocyte size-specific manner, which are coordinated with the expression of the DNMT3 family proteins. Biol Reprod 2012, 86(3):67.

50. Brown J, Jones EY, Forbes BE: Keeping IGF-II under control: lessons from the IGF-II-IGF2R crystal structure. Trends Biochem Sci 2009, 34(12):612-619.

51. Chao W, D'Amore PA: IGF2: epigenetic regulation and role in development and disease. Cytokine Growth Factor Rev 2008, 19(2):111-120. 
52. Stoger R, Kubicka P, Liu CG, Kafri T, Razin A, Cedar H, Barlow DP: Maternal-specific methylation of the imprinted mouse Igf2r locus identifies the expressed locus as carrying the imprinting signal. Cell 1993, 73(1):61-71.

53. Wutz A, Smrzka OW, Schweifer N, Schellander K, Wagner EF, Barlow DP: Imprinted expression of the Igf2r gene depends on an intronic CpG island. Nature 1997, 389(6652):745-749.

54. Barlow DP, Stoger R, Herrmann BG, Saito K, Schweifer N: The mouse insulin-like growth factor type-2 receptor is imprinted and closely linked to the Tme locus. Nature 1991, 349(6304):8487.

55. Bastepe M: The GNAS Locus: Quintessential Complex Gene Encoding Gsalpha, XLalphas, and other Imprinted Transcripts. Current genomics 2007, 8(6):398-414.

56. Plagge A, Kelsey G, Germain-Lee EL: Physiological functions of the imprinted Gnas locus and its protein variants Galpha(s) and XLalpha(s) in human and mouse. The Journal of endocrinology 2008, 196(2):193-214.

57. Seifert R, Wenzel-Seifert K, Lee TW, Gether U, Sanders-Bush E, Kobilka BK: Different effects of Gsalpha splice variants on beta2-adrenoreceptor-mediated signaling. The Beta2adrenoreceptor coupled to the long splice variant of Gsalpha has properties of a constitutively active receptor. J Biol Chem 1998, 273(18):5109-5116.

58. He Q, Zhu Y, Corbin BA, Plagge A, Bastepe M: The G protein alpha subunit variant XLalphas promotes inositol 1,4,5-trisphosphate signaling and mediates the renal actions of parathyroid hormone in vivo. Science signaling 2015, 8(391):ra84.

59. Aydin C, Aytan N, Mahon MJ, Tawfeek HA, Kowall NW, Dedeoglu A, Bastepe M: Extralarge $\mathrm{XL}(\mathrm{alpha}) \mathrm{s}$ (XXL(alpha)s), a variant of stimulatory $\mathrm{G}$ protein alpha-subunit (Gs(alpha)), is a distinct, membrane-anchored GNAS product that can mimic Gs(alpha). Endocrinology 2009, 150(8):3567-3575.

60. Weinstein LS, Yu S, Warner DR, Liu J: Endocrine manifestations of stimulatory G protein alphasubunit mutations and the role of genomic imprinting. Endocr Rev 2001, 22(5):675-705.

61. Sikora KM, Magee DA, Berkowicz EW, Berry DP, Howard DJ, Mullen MP, Evans RD, Machugh DE, Spillane C: DNA sequence polymorphisms within the bovine guanine nucleotide-binding protein Gs subunit alpha (Gsalpha)-encoding (GNAS) genomic imprinting domain are associated with performance traits. BMC genetics 2011, 12:4.

62. Chen Z, Hagen DE, Wang J, Elsik CG, Ji T, Siqueira LG, Hansen PJ, Rivera RM: Global assessment of imprinted gene expression in the bovine conceptus by next generation sequencing. Epigenetics 2016, 11(7):501-516.

63. He H, Kim J: Regulation and function of the peg3 imprinted domain. Genomics \& informatics 2014, 12(3):105-113. 
64. Relaix F, Weng X, Marazzi G, Yang E, Copeland N, Jenkins N, Spence SE, Sassoon D: Pw1, a novel zinc finger gene implicated in the myogenic and neuronal lineages. Dev Biol 1996, 177(2):383396.

65. Li L, Keverne EB, Aparicio SA, Ishino F, Barton SC, Surani MA: Regulation of maternal behavior and offspring growth by paternally expressed Peg3. Science 1999, 284(5412):330-333.

66. Rault JL, van den Munkhof M, Buisman-Pijlman FTA: Oxytocin as an Indicator of Psychological and Social Well-Being in Domesticated Animals: A Critical review. Frontiers in Psychology 2017, 8:1521-1531.

67. Huang JM, Kim J: DNA methylation analysis of the mammalian PEG3 imprinted domain. Gene 2009, 442(1-2):18-25.

68. Kaneko-Ishino T, Kuroiwa Y, Miyoshi N, Kohda T, Suzuki R, Yokoyama M, Viville S, Barton SC, Ishino F, Surani MA: Peg1/Mest imprinted gene on chromosome 6 identified by cDNA subtraction hybridization. Nat Genet 1995, 11(1):52-59.

69. Lefebvre L, Viville S, Barton SC, Ishino F, Surani MA: Genomic structure and parent-of-originspecific methylation of Peg1. Hum Mol Genet 1997, 6(11):1907-1915.

70. Tveden-Nyborg PY, Alexopoulos NI, Cooney MA, French AJ, Tecirlioglu RT, Holland MK, Thomsen $\mathrm{PD}, \mathrm{D}^{\prime}$ Cruz NT: Analysis of the expression of putatively imprinted genes in bovine periimplantation embryos. Theriogenology 2008, 70(7):1119-1128.

71. Lefebvre L, Viville S, Barton SC, Ishino F, Keverne EB, Surani MA: Abnormal maternal behaviour and growth retardation associated with loss of the imprinted gene Mest. Nat Genet 1998, 20(2):163-169.

72. Nakabayashi K, Bentley L, Hitchins MP, Mitsuya K, Meguro M, Minagawa S, Bamforth JS, Stanier $P$, Preece $M$, Weksberg $R$ et al: Identification and characterization of an imprinted antisense RNA (MESTIT1) in the human MEST locus on chromosome 7q32. Hum Mol Genet 2002, 11(15):1743-1756.

73. Joseph R, Dou D, Tsang W: Molecular cloning of a novel mRNA (neuronatin) that is highly expressed in neonatal mammalian brain. Biochem Biophys Res Commun 1994, 201(3):12271234.

74. Kagitani F, Kuroiwa Y, Wakana S, Shiroishi T, Miyoshi N, Kobayashi S, Nishida M, Kohda T, Kaneko-Ishino T, Ishino F: Peg5/Neuronatin is an imprinted gene located on sub-distal chromosome 2 in the mouse. Nucleic Acids Res 1997, 25(17):3428-3432.

75. Evans HK, Wylie AA, Murphy SK, Jirtle RL: The neuronatin gene resides in a "micro-imprinted" domain on human chromosome 20q11.2. Genomics 2001, 77(1-2):99-104.

76. Kelsey G, Bodle D, Miller HJ, Beechey CV, Coombes C, Peters J, Williamson CM: Identification of imprinted loci by methylation-sensitive representational difference analysis: application to mouse distal chromosome 2. Genomics 1999, 62(2):129-138. 
77. Zaitoun I, Khatib H: Assessment of genomic imprinting of SLC38A4, NNAT, NAP1L5, and H19 in cattle. BMC genetics 2006, 7:49.

78. Charlier C, Segers K, Wagenaar D, Karim L, Berghmans S, Jaillon O, Shay T, Weissenbach J, Cockett N, Gyapay G et al: Human-ovine comparative sequencing of a 250-kb imprinted domain encompassing the callipyge (clpg) locus and identification of six imprinted transcripts: DLK1, DAT, GTL2, PEG11, antiPEG11, and MEG8. Genome Res 2001, 11(5):850-862.

79. Wang $X$, Lan X, Radunz AE, Khatib H: Maternal nutrition during pregnancy is associated with differential expression of imprinted genes and DNA methyltranfereases in muscle of beef cattle offspring. J Anim Sci 2015, 93(1):35-40.

80. Hou XH, Li DJ, Su H, Hu JQ, Li N, Li SJ: Molecular cloning, expression, and imprinting status of maternally expressed gene 8 (Meg8) in dairy cattle. Genetika 2011, 47(8):1120-1125.

81. Nakatsu F, Messa M, Nandez R, Czapla H, Zou Y, Strittmatter SM, De Camilli P: Sac2/INPP5F is an inositol 4-phosphatase that functions in the endocytic pathway. The Journal of cell biology 2015, 209(1):85-95.

82. Choi JD, Underkoffler LA, Wood AJ, Collins JN, Williams PT, Golden JA, Schuster EF, Jr., Loomes $\mathrm{KM}$, Oakey RJ: A novel variant of Inpp5f is imprinted in brain, and its expression is correlated with differential methylation of an internal CpG island. Mol Cell Biol 2005, 25(13):5514-5522.

83. Monk D, Arnaud P, Frost JM, Wood AJ, Cowley M, Martin-Trujillo A, Guillaumet-Adkins A, Iglesias Platas I, Camprubi C, Bourc'his D et al: Human imprinted retrogenes exhibit noncanonical imprint chromatin signatures and reside in non-imprinted host genes. Nucleic Acids Res 2011, 39(11):4577-4586.

84. Bird AP: DNA methylation and the frequency of CpG in animal DNA. Nucleic Acids Res 1980, 8(7):1499-1504.

85. Kent WJ: BLAT--the BLAST-like alignment tool. Genome Res 2002, 12(4):656-664.

86. Zweig AS, Karolchik D, Kuhn RM, Haussler D, Kent WJ: UCSC genome browser tutorial. Genomics 2008, 92(2):75-84.

87. Lee CM, Barber GP, Casper J, Clawson H, Diekhans M, Gonzalez JN, Hinrichs AS, Lee BT, Nassar LR, Powell CC et al: UCSC Genome Browser enters 20th year. Nucleic Acids Res 2020, 48(D1):D756-D761.

88. Bina M: The genome browser at UCSC for locating genes, and much more! Molecular biotechnology 2008, 38(3):269-275.

89. Haeussler M, Zweig AS, Tyner C, Speir ML, Rosenbloom KR, Raney BJ, Lee CM, Lee BT, Hinrichs AS, Gonzalez JN et al: The UCSC Genome Browser database: 2019 update. Nucleic Acids Res 2019, 47(D1):D853-D858. 
bioRxiv preprint doi: https://doi.org/10.1101/2021.12.27.474271; this version posted December 28, 2021. The copyright holder for this preprint (which was not certified by peer review) is the author/funder. All rights reserved. No reuse allowed without permission. 